\title{
Polipose Múltipla Familiar. Análise de 44 Casos Tratados no Hospital das Clínicas da FMRP-USP
}

\author{
Familiar Adenomatosis Polyposis: Analysis Of Forty-Four Cases from the School \\ of Medicine of Riberão Preto Hospital and Clinics
}

\author{
ANDREZAREGINADE B. M. DASILVA'; ROGÉRIO SERAFIM PARRA ${ }^{1}$; JAICER GONÇALVES ROLO ${ }^{2}$; ROBERTO \\ BUENO FILHO²; OMAR FÉRES 3 ; JOSÉ JOAQUIM RIBEIRO DA ROCHA ${ }^{3}$ \\ ${ }^{1}$ Pós-graduado da Área de Clínica Cirúrgica do Depto. de Cirurgia e Anatomia, FMRP-USP, ${ }^{2}$ Aluno de graduação da \\ FMRP-USP; ${ }^{3}$ Professor Doutor da Disciplina de Coloproctologia, Depto. Cirurgia e Anatomia, FMRP-USP.
}

SILVA ARBM; PARRA RS; ROLO JG; BUENO FILHO R; FÉRES O; ROCHA JJR. Polipose Múltipla Familiar. Análise de 44 Casos Tratados no Hospital das Clínicas da FMRP-USP. Rev bras Coloproct, 2007;27(3): 310-316.

RESUMO: Os autores apresentam análise retrospectiva de 44 pacientes com Polipose Múltipla Familiar tratados no Hospital das Clínicas da Faculdade de Medicina de Ribeirão Preto,Universidade de São Paulo, entre janeiro de 1991 a julho de 2005. Foram estudados aspectos epidemiológicos (idade, sexo), genéticos, principais sintomas, antecedentes pessoais e aspectos envolvendo tratamento cirúrgico e complicações pós-operatórias, comparando os achados com os da literatura correlata. Dos pacientes, 31 são do sexo masculino e 13 do feminino com idade média de 32 anos (14 - 60 anos). Os sintomas prevalentes foram: sangramento intestinal (62,5\%), alteração do hábito intestinal (60\%), e com menor freqüência dor abdominal (45 \%) e emagrecimento (30\%). Relataram casos de polipose familiar $67,5 \%$ dos pacientes e $62,5 \%$ referiram parentes com antecedente de neoplasias (intestinal e extra-intestinal). Cerca de $32,5 \%$ dos pacientes já apresentavam neoplasia de cólon na época do diagnóstico da polipose,com idade média de 39 anos. $O$ tratamento cirúrgico foi realizado em $95,4 \%$ dos pacientes: $35,7 \%$ foram submetidos à proctocolectomia total $(9$ casos com bolsa ileal em $J$ e 6 casos com ileostomia definitiva) e 59,2\% a colectomia total com ileorretoanastomose. Atualmente $57 \%$ dos pacientes avaliados ainda estão em seguimento com reavaliações periódicas, $7 \%$ faleceram e $27 \%$ abandonaram o tratamento.

Descritores: polipose familiar, câncer colorretal, colectomia, genética.

\section{INTRODUÇÃo}

A polipose adenomatosa familiar (PAF) é uma doença hereditária , autossômica dominante ,com penetrância próxima de $100 \%$, causada por uma mutação no Adenomatous polyposis coli (APC), localizado no cromossomo $5 \mathrm{q} 21$. APC é um gene supressor de tumor, identificado em 1987, e reproduzido em 1991 após análise de mutações em famílias com PAF $^{1}$.

Sua incidência é de aproximadamente $1 \mathrm{em}$ 7000 a 1 em 16000 nascidos vivos, sendo responsável por menos de $1 \%$ dos casos de câncer colorretal ${ }^{2}$.
Mutações no APC são os passos iniciais para o desenvolvimento do câncer colorretal. Ele está relacionado com atividades celulares fundamentais nos processos de produção de proteínas, adesão celular e migração. Quando o APC sofre mutação, há interferência na proliferação, apoptose e controle das mudanças de fase do ciclo celular ${ }^{3}$.

A identificação de alterações no gene APC é feita com auxílio de testes genéticos e apesar disto, 20 a $30 \%$ dos pacientes com PAF não apresentam alterações do gene no teste de rastreamento genético. Nestes casos testes de maior sensibilidade, como o MAMA

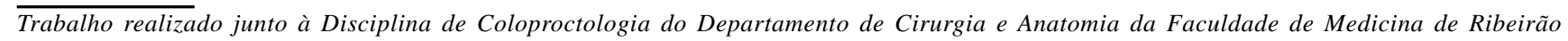
Preto-USP (FMRP-USP). 
Rev bras Coloproct Julho/Setembro, 2007 (monoallelic mutation analysis) podem ser usados. A combinação destes dois testes permite que em $95 \%$ dos casos as alterações do gene APC sejam identificadas ${ }^{4}$.

A PAF se manifesta pela presença de numerosos pólipos adenomatosos em todo trato gastrointestinal, principalmente o cólon. Estes pólipos podem estar presentes vários anos antes do aparecimento dos sintomas, fato que ratifica a importâncias de investigação precoce dos familiares de pacientes acometidos pela doença. Estudos têm afirmado a existências de pólipos por pelo menos uma década antes do aparecimento dos sintomas ${ }^{1}$. Estes pólipos progridem para câncer colorretal até 35-40 anos se não tratados adequadamente.

Os pólipos também acometem o trato gastrointestinal alto (estômago e duodeno) sendo que aproximadamente $2 / 3$ dos adenomas duodenais ocorrem na região periampular ${ }^{5}$. Adenomas duodenais avançados representam risco aumentado de câncer, sendo a terceira causa de morte em pacientes com polipose adenomatosa familiar $(8,2 \%)$, ficando atrás das metástases do CCR $(58,2 \%)$ e de tumores desmóides $(10,9 \%)^{6}$.

Outra forma de apresentação clínica é a hipertrofia congênita do epitélio pigmentado da retina (CHRPE), que se caracteriza pela presença de lesões pigmentadas no exame de fundo de olho ocorrendo em 70-80\% dos pacientes com polipose. Estas alterações oftalmológicas estão usualmente presentes no nascimento, são assintomáticas, sem potencial maligno e podem ser avaliadas por exames de baixa complexidade ${ }^{7}$.

Tumores desmóides são mais raros, ocorrendo em 15\% dos casos de $\mathrm{PAF}^{8}$, mais freqüentemente no abdome e parede abdominal (50\%). Preferencialmente são assintomáticos e lesões intraabdominais podem causar dor ou podem ser complicadas por obstrução ureteral, hemorragias e até mesmo fístulas enterocutâneas 9 .

O câncer da tireóide ocorre em 1 a $2 \%$ dos casos. Tendem a ser bem delimitados, pouco agressivos e com baixo potencial de metástases ${ }^{10,11}$.

A associação de pólipos gastrointestinais com osteomas caracteriza uma das variantes da PAF , Síndrome de Gardner. Osteomas tipicamente ocorrem na mandíbula e ossos longos. São tumores benignos e indolores, e normalmente seu diagnóstico precede o da polipose ${ }^{12}$.
A síndrome de Turcot é a associação da PAF com tumores primários do Sistema Nervoso Central , sendo mais comum o meduloblastoma ${ }^{13}$.

Polipose atenuada é uma variante fenotípica distinta que se caracteriza pela presença de menos de 100 pólipos colônicos e localizados preferencialmente no cólon direito. Nestes casos o desenvolvimento do câncer colorretal é mais tardio (15 anos mais tarde do que nos casos clássicos) ${ }^{14}$.

\section{OBJETIVO}

O objetivo deste trabalho é estudar 44 pacientes com diagnóstico de polipose adenomatosa familiar, analisando aspectos epidemiológicos, genéticos , principais sintomas, antecedentes pessoais e o tipo de tratamento proposto, avaliando também suas complicações.

\section{MATERIAL E MÉTODOS}

Foi realizada avaliação retrospectiva do prontuário de 44 pacientes acompanhados no Serviço de Coloproctologia do HCRP-USP no período de janeiro de 1991 a julho de 2005,obtendo-se os dados a partir de um protocolo pré-elaborado. Os seguintes dados foram revisados: prevalência de sexo e idade, principais sintomas apresentados, alterações no exame físico, neoplasias associadas, antecedentes familiares de polipose colônica e neoplasias. Também foi revisado o tipo de tratamento cirúrgico e suas complicações pósoperatórias, e fatores relacionados a qualidade de vida pós-operatória como a presença de incontinência fecal, necessidade do uso de medicamentos antidiarréicos e o hábito intestinal após a cirurgia.

Após a coleta de dados, foi realizada a análise da freqüência simples das variáveis correlacionando os achados com os da literatura correlata.

\section{RESULTADOS}

Foram avaliados 44 pacientes, sendo 31 do sexo masculino e 13 do feminino,com idade média de 32 anos (14-60 anos) (Tabela 1). Os sintomas prevalentes foram sangramento intestinal $(62,5 \%)$, alteração do hábito intestinal (60\%), e com menor freqüência dor abdominal (45\%) e emagrecimento (30\%).

Dos pacientes, $67,5 \%$ relataram polipose familiar, sendo que $62,5 \%$ referiram parentes com ante- 
Tabela 1 - Distribuição dos pacientes segundo o sexo e idade.

\begin{tabular}{lcccccc}
\hline Sexo & \multicolumn{7}{c}{ Idade (anos) } \\
\cline { 2 - 7 } & $\mathbf{1 0 - 2 0}$ & $\mathbf{2 0 - 3 0}$ & $\mathbf{3 0 - 4 0}$ & $\mathbf{4 0 - 5 0}$ & $\mathbf{5 0 - 6 0}$ & Total \\
Masculino & 7 & 6 & 4 & 9 & 4 & 31 \\
Feminino & 4 & 6 & 1 & 2 & 1 & 13 \\
Total & 11 & 12 & 5 & 11 & 5 & 44 \\
\hline
\end{tabular}

cedente de neoplasias (intestinal e extra-intestinal). Cerca de $32,5 \%$ dos pacientes já apresentavam neoplasia de cólon na época do diagnóstico da polipose, com idade média de 39 anos (Fig. 1,A,B). Alterações no exame de endoscopia digestiva alta ocorreram em $11,3 \%$ dos pacientes.

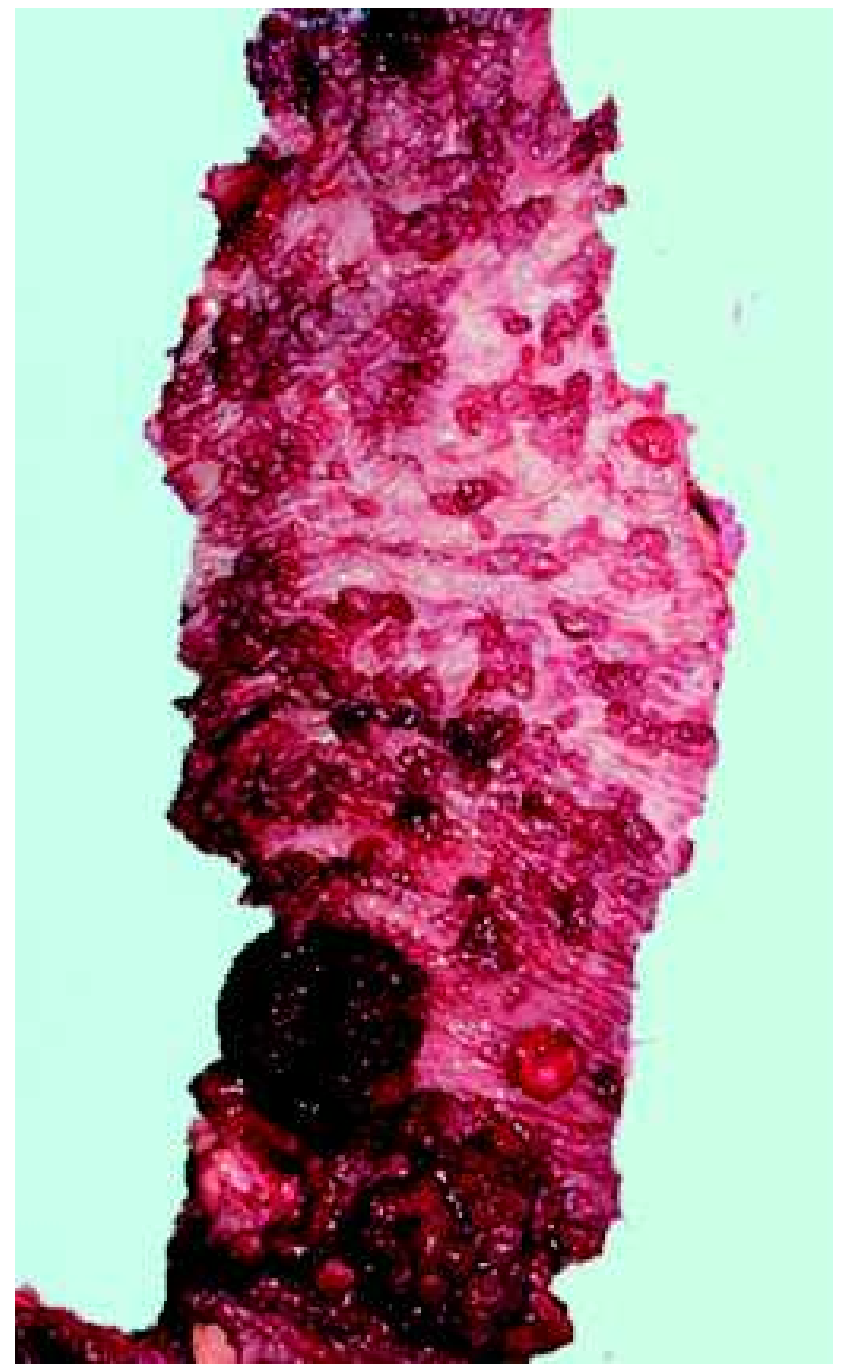

Figura 1A - Cólon acometido por polipose e com neoplasia.
O tratamento cirúrgico foi realizado em 95,4\% dos pacientes: $35,7 \%$ foram submetidos à proctocolectomia total ( 9 casos com bolsa ileal em J e 6 casos com ileostomia definitiva) e 59,2\% a colectomia com ileorretoanastomose. No pós-operatório $33 \%$ dos pacientes apresentaram complicações precoces e $24 \%$ complicações tardias (Tabela 2). No seguimento, 4,5\% dos pacientes evoluíram com 5 ou mais evacuações ao dia necessitando de medicamentos antidiarreicos e 4\% mantiveram queixa de incontinência fecal. Atualmente $57 \%$ dos pacientes avaliados ainda estão em seguimento com reavaliações periódicas, $7 \%$ faleceram e $27 \%$ abandonaram o tratamento.

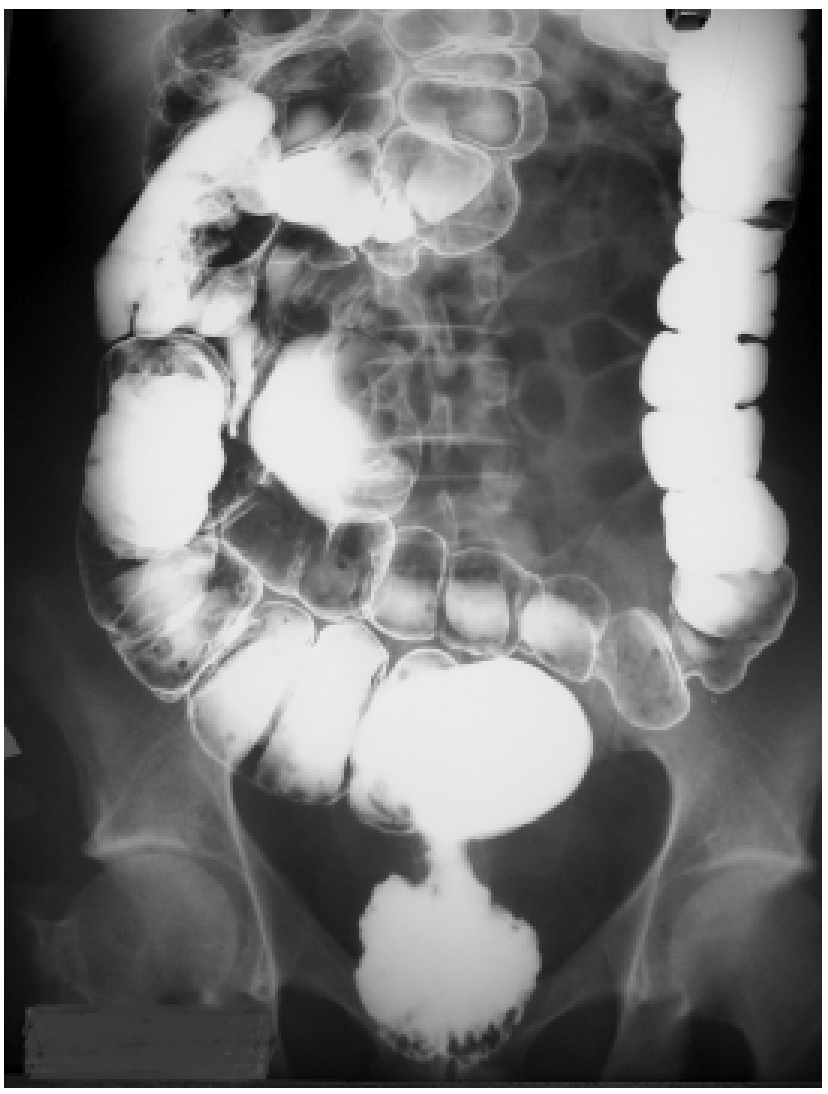

Figura $1 B$ - Enema opaco evidenciando neoplasia estenosante do reto. 
Rev bras Coloproct Julho/Setembro, 2007
Polipose Múltipla Familiar. Análise de 44 Casos Tratados

no Hospital das Clínicas da FMRP-USP

Vol. 27

Andreza Regina de B. M. da Silva e Cols.

Tabela 2 - Complicações precoces e tardias do tratamento cirúrgico.

\begin{tabular}{lrlc}
\hline \multicolumn{3}{c}{ COMPLICAÇÕES } & $(\boldsymbol{\%})$ \\
\hline Precoces & $(\boldsymbol{\%})$ & Tardias & 11 \\
\hline Abscesso & 11 & Obstrução intestinial & 4,5 \\
Deiscência & 9 & Ejaculação retrógrada & 4,5 \\
Lesões da parede & 9 & Diarréia & 2 \\
Pancreatite & 2 & Estenose da anastomose & 2 \\
Sangramento & 2 & TVP & \\
\hline
\end{tabular}

\section{DISCUSSÃO}

A análise dos dados mostrou que a polipose adenomatosa familiar é doença de caráter hereditário(67,5\% dos pacientes relataram antecedentes familiares), que acomete indivíduos jovens (idade média de 32 anos) e embora tenha havido maior número de casos em pacientes do sexo masculino, não encontramos na literatura descrição de prevalência entre os sexos , ou seja , é doença igualmente distribuída entre ambos os sexos.

O principal sintoma apresentado foi o sangramento intestinal ocorrendo em $62,5 \%$ dos casos , número inferior ao descrito na literatura que é cerca de $80 \%$,seguido pela alteração do hábito intestinal $(60 \%)$ e dor abdominal (45\%) .

Além da avaliação quanto a casos de parentes com diagnóstico ou já tratados de polipose adenomatosa familiar, também avaliamos a presença de outros antecedentes, como a informação sobre algum familiar tratado ou que faleceu após o diagnóstico de neoplasias (intestinais e extraintestinais). Souberam referir sobre outros casos de neoplasia $62,5 \%$ dos pacientes.

Dos 44 pacientes avaliados, $32,5 \%$ já apresentavam neoplasia colorretal quando diagnosticada a presença de polipose colônica, com idade média de 39 anos o que reforça o fato de que se não tratados adequadamente até os 40 anos ocorrerá degeneração e malignização dos adenomas (Fig. 2). O rastreamento de pacientes e familiares de risco é importante, pois tem reduzido em $55 \%$ a ocorrência do câncer colorretal no momento do diagnóstico da polipose, e representa aumento de sobrevida dos pacientes tratados ${ }^{18,19}$.

Todos os pacientes foram encaminhados para o setor de genética do HCRP, onde foram submetidos à confecção de heredograma, para melhor identificação dos acometidos, e aconselhamento genético (Fig. 3).

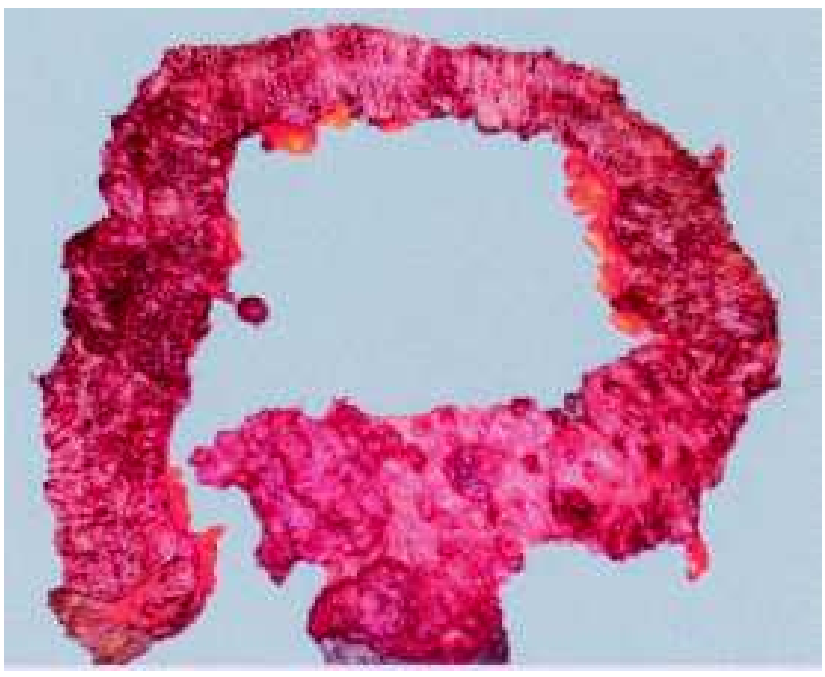

Figura 2 - Cólon acometido por polipose.

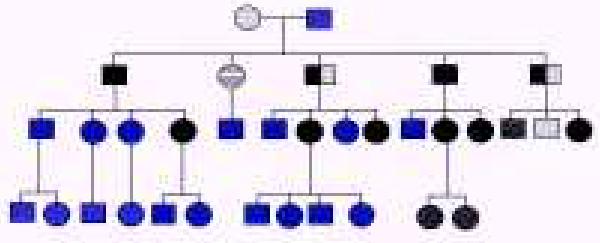

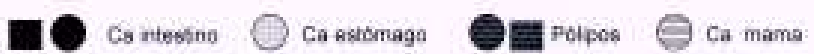

Figura 3 - Heredograma demonstrando indivíduos acometidos.

Atualmente, é possível, através de teste genético específico, detectar quais os portadores de defeito genético predisponente para FAP dentro de uma mesma família. Para isso, uma pessoa sabidamente portadora da doença deve colher sangue, que será testado por técnicas altamente especializadas (seqüenciamento genético automático computadorizado), visando localizar dentro do 
Rev bras Coloproct Julho/Setembro, 2007 gene APC defeituoso, o problema específico para aquela determinada família. Encontrado o defeito genético predisponente da FAP no paciente em questão, outros membros da família, sem a doença manifestada, podem ter seu sangue testado com a finalidade de pesquisar, direta e especificamente, se têm a mesma predisposição. Os resultados são confidenciais, entregues em mãos, pelo médico, diretamente ao paciente.

AAssociação Americana de Gastroenterologia recomenda sigmoidoscopia anual, iniciando aos 10-12 anos para pacientes com diagnóstico genético de polipose e para membros de família de risco que não dispõe de testes genéticos (15), como se enquadram os pacientes desta análise.

A colectomia é o tratamento recomendado para reduzir o risco de câncer corretal em pacientes com polipose adenomatosa familiar. As opções cirúrgicas incluem colectomia total com ileorretoanastomose , proctocolectomia total com ileostomia terminal ou com bolsa ileal em J . Em nossa revisão 96\% dos pacientes foram submetidos ao tratamento cirúrgico, sendo $60,4 \%$ dos pacientes submetidos à colectomia total com ileorretoanastomose , 20,9\% a proctocolectomia total com bolsa ileal em J e 13,9\% ficaram com ileostomia definitiva após a proctocolectomia (Tabela 3) . Quando analisados os dados com relação ao passar dos anos notamos um aumento substancial dos casos de proctocolectomia total com bolsa ileal em J, embora no contexto total ainda prevaleça a colectomia total com ileorretoanastomose. Isso vem ocorrendo devido a maior facilidade técnica de equipe, e aquisição de grampeadores que reduziram em muito o tempo cirúrgico para confecção dos reservatórios.

A colectomia com ileorretoanastomose é tecnicamente mais simples, tradicionalmente associada com menores índices de complicações pós-operatórias e melhores resultados funcionais , mas tem se tornado uma técnica menos atrativa, pois mantém o risco do câncer devido a presença da mucosa retal. O risco cumulativo estimado é de $10 \%$ aos 50 anos de vida, aumentando para $29 \%$ aos $60 \operatorname{anos}^{16}$.

Nos pacientes submetidos a proctocolectomia total com bolsa ileal em J somente em 1 caso usamos a mucosectomia com anastomose ileoanal manual. Em todos os demais pacientes optamos pelo duplo grampeamento com stappler. O duplo grampeamento tem se mostrado uma técnica segura, embora exista a possibilidade da permanência de $1-2 \mathrm{~cm}$ de mucosa retal residual favorecendo o desenvolvimento de adenoma / carcinoma ${ }^{17}$.

Na avaliação pós-operatória pacientes submetidos a colectomia total com ileorretoanastomose apresentavam hábito intestinal de 2 a 3 evacuações / dia enquanto aqueles submetidos à proctocolectomia total com bolsa ileal em J, apresentavam 4 a 5 evacuações/ dia. Não foram relatados restrições alimentares ou sociais, e $2 \%$ dos pacientes queixaram-se de disfunção sexual. Mesmo após todo período de adaptação, 6,8\% dos pacientes mantiveram o uso de medicamentos antidiarréicos e $2 \%$ mantiveram queixa de incontinência fecal. Os estudos da literatura,mostram que independente da técnica cirúrgica utilizada não há diferença significativa entre os grupos para a presença de incontinência ou necessidade do uso de medicamentos antidiarréicos ${ }^{18,19}$.

\section{CONCLUSÃO}

A polipose adenomatosa familiar é doença hereditária que se manifesta em indivíduos jovens, e se não tratados adequadamente desenvolverão neoplasia colorretal.

As famílias portadoras de FAP devem ser registradas, ou seja, catalogadas, com construção de heredograma (árvore genealógica) e de preferência com identificação do defeito genético específico

Tabela 3 - Tipos de cirurgias realizadas e idade dos pacientes.

\begin{tabular}{lrrrrr}
\hline & \multicolumn{5}{c}{ Idade (anos) } \\
\cline { 2 - 6 } Cirurgias & $\mathbf{1 0 - 2 0}$ & $\mathbf{2 0 - 3 0}$ & $\mathbf{3 0 - 4 0}$ & $\mathbf{4 0 - 5 0}$ & $\mathbf{5 0 - 6 0}$ \\
\hline Ileoretoanastomose & 11 & 4 & 4 & 5 & 2 \\
Ileostomia definitiva & - & 3 & - & 2 & 1 \\
Bolsa ileal em “j” & - & 2 & - & 6 & 1 \\
Total & 11 & 9 & 4 & 14 & 4 \\
\hline
\end{tabular}


Rev bras Coloproct Julho/Setembro, 2007
Polipose Múltipla Familiar. Análise de 44 Casos Tratados no Hospital das Clínicas da FMRP-USP Andreza Regina de B. M. da Silva e Cols. predisponente da doença. A família deve ser orientada detalhadamente quanto às características da doença, inclusive das chances de transmissão para os filhos, da possibilidade de teste genético de predisposição, das opções de tratamento e de acompanhamento. Deve ser oferecido todo o apoio profissional da equipe em cada fase do tratamento. $\mathrm{O}$ melhor tratamento continua a ser o tratamento cirúrgico. Diversos estudos tem indicado que o ideal para reduzir o risco do câncer colorretal é realizar cirurgia mais ampla, com ressecção total da mucosa colônica com bolsa ileal em $\mathrm{J}$.

\begin{abstract}
The authors present a retrospective analysis of forty-four patients with familiar adenomatosis polyposis treated at the School of Medicine of Ribeirão Preto Hospital and Clinics - University of São Paulo, from January 1991 to July 2005. Epidemiologic (age and gender) and genetic aspects were investigated as well as main symptoms, personal history and surgical treatment outcome. Data obtained were compared to the available literature. Our results show that 31 patients were male and 13 female, with average age of 32 years-old (14 to 60 years-old). The main symptoms were intestinal bleeding (62.5\%) and intestinal habit alteration $(60 \%)$. Less frequently, patients showed abdominal pain (45 \%) and loss of weight (30 \%). Familiar adenomatosis polyposis was reported by $67.5 \%$ of the patients and relatives with neoplasms (intestinal and extra-intestinal) were referred by $62.5 \%$ of the patients. By the time of the polyposis diagnosis, $32.5 \%$ of the patients have already been diagnosed for colon cancer, with average age of 39 years old. Surgical treatment was performed in $95.4 \%$ of the patients, total proctocolectomy was performed in $35.7 \%$ ( 9 cases with ileal J- pouch and 6 with permanent ileostomy) and total colectomy with ileo rectal anasthomosis in $59.2 \%$. From all patients evaluated, $57 \%$ are still under follow up, $7 \%$ died and $27 \%$ gave up treatment.
\end{abstract}

Key words: familiar polyposis, colorectal cancer, colectomy, genetics.

\section{REFERÊNCIAS}

1. Groden J, Thliveris A, Samowitz W, et al. Identification and characterization of the familial adenomatous polyposis coli gene. Cell 1991; 66: 589-600.

2. Burt RW, Bishop DT, Lynch HT, et al. Risk and surveillance of individuals with heritable factors for colorectal cancer. WHO Collaborating Centre for the Prevention of Colorectal Cancer. Bull World Health Organ 1990; 68: 655-65.

3. Näthke I. APC at a glance. J Cell Sci 2004;117: 4873-5

4. Laken SJ, Papadopoulos N, Petersen GM, et al. Analysis of masked mutations in familial adenomatous polyposis. Proc Natl Acad Sci USA 1999; 96: 2322-6.

5. Bertoni G, Sassateli R, Nigrisoli E, et al. High prevalence of adenomas and microadenomas of the duodenal papilla and periampulllary region in patients with familial adenomatous polyposis. Eur J Gastroenterol Hepatol 1996; 8: 1201-6.

6. Arvantis ML, Jagelman DG,Fazio VW, et al. Mortality in patients with familial adenomatous polyposis Dis Colon Rectum 1990; 33: 639-42

7. Trabulsi El, Krush AJ, Gardner EJ, et al . Prevalence and importance of pigmented ocular fundus lesions in Gardner's syndrome. N Engl J Med 1987; 316: 661-7.

8. Sturt NJH, Gallagher MC, Bassett P, et al. Evidence for genetic predisposition to desmoid tumours in familial adenomatous polyposis independent of the germline APC mutation. Gut 2004; 53: 1832-6.

9. Clark SK, Neale KF, Landgrebe JC, et al. Desmoids tumours complicating familial adenomatous polyposis. Br J Surg 1999; 86: $1185-9$
10. Bülow C, Bülow S, Leeds Castle Polyposis Group . Is screening for thyroid carcinoma indicated in familial adenomatous polyposis? Int J Colorectal Dis 1997; 12: 2402.

11. Perrier ND, Van Heerden JA, Goellner JR, et al. Thyroid cancer in patients with familial adenomatous polyposis. World J Surg 1998; 22: 738-42.

12. Bilkay U, Erdem O, Ozek C, et al. Benign osteoma with Gardner syndrome: Review of the literature and report of a case. J Craniofac Surg 2004; 15: 506-9.

13. Hamilton SR, Liu B, Parsons RE, et al. The molecular basis of Turcot's Syndrome. N Engl J Med 1995; 332: 839-47.

14. Burt RW, Leppert MF, Slattery ML, et al. Genetic testing and phenotype in a large kindred with attenuated familial adenomatous polyposis. Gastroentorology2004; 127: 44451.

15. Winawer S, Fetcher R, Rex D, et al. (U. S. Multisociety Task Forece on Colorectal Cancer) Colorectal cancer screening and surveillance: Clinical guidelines and rationale - Update based on new evidence. Gastroenterology2003; 124: 54460 .

16. Nugent KP, Phillips RK. Rectal cancer risk in older patients with familial adenomatous polyposis and an ileorectal anastomosis: A cause for concern. Br J Surg 1992;1204-6.

17. Ziv Y, Fazio VW, Church JM, Lavery IC, King TM, Ambrosette p. Stapled ileal pouch anal anastomoses are safer than handsewn anastomoses in patients with ulcerative colitis. Am J Surg 1996; 171: 320-3.

18. Rossi BM, Nagakawa, WT, Oliveira FO, Aguiar Jr S, Lopes a Câncer de cólon reto e ânus. Tecmed Ed. São Paulo, 2005. 
19. Rossi BM, Corvello CM, Anelei A, Epilman C, Paegle LD, Nagakawa WT, Borghes AY, Amorim C, Duarte APM, Simpson AJG, Lopes ${ }^{a}$ Hereditary colorectal tumor: routine case and multidisciplinary therapeutic approach. South Am J Câncer 1997; 1: 191-7
Endereço para correspondência: PROF. DR. OMAR FERES

Departamento de Cirurgia e Anatomia - Disciplina de Coloproctologia

Hospital das Clínicas da FMRP-USP

email: marlenelucio@yahoo.com.br / feresomar@netsite.com.br CAMPUS UNIVERSITÁRIO

14.048-900 